\title{
ORIGINAL
}

\section{BACTERIEMIAS POR STAPHYLOCOCCUS AUREUS: FACTORES DE RIESGO ASOCIADOS A LA RESISTENCIA A METICILINA}

${ }^{1}$ Medico especialista en Microbiología.

${ }^{2}$ Medico especialista en Medicina Preventiva y Salud Pública.

aservicio Microbiología Hospital de Barbastro (Huesca) bServicio Medicina Preventiva Hospital de Barbastro (Huesca) cServicio Microbiología Hospital San Jorge (Huesca) dDepartamento Microbiología, Preventiva y Salud Pública Universidad Zaragoza

Correspondencia a: Nombre: Ana Betrán Correo electrónico: abetrane@ salud.aragon.es

Palabras clave: Staphylococcus aureus. Meticilina. Bacteriemia

Keywords: Staphylococcus aureus; methicillin; bacteremia

Procedencia y arbitraje: no comisionado, sometido a arbitraje externo.

Recibido para publicación 22 de noviembre de 2019 Aceptado para publicación: 13 de julio de 2020

\section{Citar como:}

Betrán A, Lapresta C, Lavilla J, Abad-Díez JM Torres L. Bacteriemias por staphylococcus aureus:factores de riesgo asociados a la resistencia a meticilina. Rev Cient Cienc Med 2020; 23(1) 44-51

\section{BACTERIEMIAS CAUSED BY STAPHYLOCOCCUS AUREUS: RISK FACTORS}

ASSOCIATED TO METHICILLIN RESISTANCE

Ana Betrán ${ }^{1, a, d}$, Carlos Lapresta ${ }^{2, b}, M^{a}$ José Lavilla ${ }^{1, c}$, José María Abad-Díez ${ }^{4, d}$, Luis Torres ${ }^{1, c}$

\section{RESUMEN}

Introducción: El objetivo del trabajo ha sido identificar los factores de riesgo que podrían favorecer la aparición de resistencia a meticilina en aislamientos de Staphylococcus aureus y aquellos que influirían en la mortalidad por las bacteriemias producidas por este patógeno.

Métodos: Se realizó un estudio observacional de casos y controles en los 57 pacientes diagnosticados de bacteriemia por Staphylococcus aureus en el Hospital de Barbastro. Para el análisis se utilizaron los test estadísticos de Chi cuadrado de Pearson, test de Fisher y regresión logística múltiple.

Resultados: Del total de bacteriemias, 63,15\% correspondieron a Staphylococcus aureus sensible a meticilina y $36,84 \%$ a Staphylococcus aureus resistente a meticilina. Se asociaron a resistencia a meticilina, la adquisición nosocomial de la infección, el uso previo de antibióticos y la edad mayor de 65 años. La mortalidad de los casos de bacteriemia por SARM y SAMS fue respectivamente del $28,57 \%$ y del 36,1 I $\%$, siendo estas diferencias estadísticamente no significativas. Todos los aislamientos fueron sensibles in vitro a vancomicina.

Conclusiones: En nuestro sector sanitario, las estrategias más efectivas para disminuir la incidencia de bacteriemias por SARM serían el control y uso adecuado de antimicrobianos y la aplicación de programas de prevención de infecciones nosocomiales. En los pacientes con bacteriemia por Staphylococcus aureus, la resistencia a meticilina no se asocia a más mortalidad.

\section{ABSTRACT}

Introduction: The objective of this work has been to identify the risk factors that could favor the appearance of methicillin resistance in isolates of Staphylococcus aureus (SA) and those that would influence mortality due to bacteremia produced by this pathogen.

Methods: An observational case-control study was carried out in 57 patients diagnosed with bacteremia by SA at the Barbastro's Hospital. Pearson Chi square statistical test, Fisher test and multiple logistic regression were used for the analysis.

Results: Of the total Bacteriemias, 63.15\% corresponded to methicillin-sensitive Staphylococcus aureus (MSSA) and $36.84 \%$ to methicillin-resistant Staphylococcus aureus (MRSA). Nosocomial acquisition of the infection, previous use of antibiotics and the age over 65 years, were associated with methicillin resistance. The mortality of cases of MRSA and SAMS bacteremia was $28.57 \%$ and $36.11 \%$ respectively, these differences being statistically not significant. All isolates were sensitive in vitro to vancomycin.

Conclusions: In our health sector, the most effective strategies to reduce the incidence of MRSA bacteremia would be the control and proper use of antimicrobials and the application of nosocomial infection prevention programs. Patients with SA bacteremia, are not associated with more mortality caused by MRSA.

\section{INTRODUCCIÓN}

taphylococcus aureus (SA) es la principal Sespecie patógena de su género, y es responsable de infecciones diversas, tanto de origen comunitario como relacionadas con el sistema sanitario'. Es uno de los microorganismos patógenos más versátiles que existen y presenta una patogenicidad variable que le permite causar desde infecciones banales hasta infecciones con compromiso vital como bacteriemias, endocarditis o meningitis ${ }^{2}$ 3 .

La introducción de la penicilina a principios de los años 40 como tratamiento de las infecciones causadas por SA, consiguió disminuir las enfermedades producidas por este microorganismo; sin embargo, un año después 
de su utilización ya se aislaban cepas de SA resistentes a este antibiótico por producción de penicilinasas ( $\beta$-lactamasas) ${ }^{4}$. El año 1946 en Inglaterra, un $60 \%$ de los aislamientos de estafilococos eran resistentes a penicilina; hoy en día se describen resistencias en el $80 \%$ 93\% de las cepas de SA, aisladas tanto a nivel comunitario como hospitalario ${ }^{5}$. Consecuencia del incremento de resistencia a la penicilina, a finales de los años 50 comenzó a usarse en Europa la meticilina, un derivado semisintético de la penicilina eficaz frente a infecciones por SA. El primer aislamiento de Staphylococcus aureus resistente a la meticilina (SARM) fue descrito en Inglaterra el año 1961, dos años después de la introducción de la meticilina en el mercado ${ }^{6}$. Desde entonces la prevalencia de SARM ha ido creciendo en la mayoría de países y hemos asistido a un incremento notabley continuo pese a la implementación de diferentes programas de control $^{7}$. Si bien el término resistencia a meticilina incluye resistencia a derivados ß-lactámicos, las cepas SARM presentan a través de diversos mecanismos resistencia múltiple a varios grupos de antibióticos, describiéndose cada vez con mayor frecuencia brotes de SARM sensibles sólo a los glucopéptidos ${ }^{8}$. Aunque la vancomicina o la teicoplanina siguen siendo el tratamiento de elección frente a una cepa SARM, en los últimos años se ha descrito un fenómeno de disminución de sensibilidad a los glucopéptidos; las primeras infecciones por SA con resistencia intermedia a glucopéptidos se documentaron en Japón ${ }^{9}$ y en Estados Unidos en los años 90, iniciándose entonces debates acerca de su significación tanto epidemiológica como clínica ${ }^{10}$. Estas cepas se denominan VISA o GISA (Vancomycin Intermediate Staphylococcus Aureus o Glycopeptide Intermediate Staphylococcus Aureus A) y se asocian a fracasos terapéuticos con glucopéptidos, lo que conduce a la necesidad detectar y controlar precozmente este tipo de aislamientos ${ }^{11}$.

En España los primeros casos de SARM se observaron en 1981 y desde entonces el porcentaje de infecciones producidas por este microorganismo fue aumentado tanto en nuestro entorno como en la mayoría de países ${ }^{12}$.

Las bacteriemias por SARM representan aproximadamente el $20-21 \%$ de las infecciones causadas por este microorganismo y el $10-50 \%$ de las bacteriemias por SA atendidas en el ámbito hospitalario ${ }^{13}$. La mortalidad asociada a la bacteriemia por SARM es superior al 30\%, pudiendo llegar al $60 \%$ en alguno de los estudios publicados ${ }^{14}$. La posibilidad de que las bacteriemias causadas por cepas SARM otorguen un peor pronóstico y una mayor mortalidad que las causadas por SAMS ha sido motivo de diferentes revisiones y en la mayoría de ellas, la mortalidad asociada a la bacteriemia por SARM suele ser superior a la asociada a SAMS.

Analizando algunos datos sobre resistencias antibióticas del Hospital de Barbastro, se observó que la prevalencia de SARM en los últimos años en nuestra área sanitaria era superior a la del resto de España publicada por el Sistema de Vigilancia Europeo. Por este motivo, se planteó realizar un estudio de las infecciones estafilocócicas, centrándonos en las bacteriemias por SARM ya que se asocian a una elevada morbimortalidad y además poseen pocas alternativas de tratamiento antibiótico. También se consideró interesante analizar los factores de riesgo habitualmente asociados con la adquisición de resistencia a meticilina, lo que podría ayudarnos a adoptar estrategias de prevención y así disminuir la incidencia de estas infecciones en nuestro Sector Sanitario.

Por lo expuesto anteriormente, el objetivo de este trabajo ha sido doble: por un lado, identificar los factores de riesgo que pueden favorecer la aparición de resistencia a meticilina en aislamientos de SA y en segundo lugar, analizar si la resistencia a meticilina incrementa la mortalidad en pacientes con bacteriemia por SA.

\section{MATERIALES Y MÉTODOS}

Se realizó un estudio observacional, de tipo analítico de casos y controles. Se incluyeron todos los pacientes con diagnóstico de bacteriemia por SA en el periodo de julio de 2010 a diciembre de 2014 y se consideró como casos expuestos; los pacientes que presentaron bacteriemias por SARM y como controles expuestos; los pacientes con bacteriemias por SAMS.

El Hospital de Barbastro dispone de 160 camas en el área de hospitalización y 5 quirófanos en el área quirúrgica. Los servicios clínicos en que ingresaban los pacientes se clasificaron en tres grupos: Servicios Médicos (Medicina Interna, Especialidades Médicas y Urgencias), Servicios 
Quirúrgicos (Cirugía y otras Especialidades Quirúrgicas) y Servicios de "alto riesgo" (UCl, Hematología y Oncología).

Se recogieron datos de las historias clínicas de los 57 pacientes diagnosticados de bacteriemia por SA en nuestro hospital. Las variables estudiadas fueron: edad (con dos categorías: $\geq 65$ años y $<65$ años), sexo, mortalidad (si el motivo de alta del paciente en ese ingreso fue por fallecimiento), enfermedades de base presentes en el momento del diagnóstico de bacteriemia (diabetes, neoplasia, enfermedad pulmonar obstructiva crónica, insuficiencia renal), factores de riesgo en el momento del diagnóstico de sepsis (portador de catéter central, cirugía, antibioterapia previa, ingresos en el último año), presencia o no de algún foco de infección en el momento del diagnóstico de bacteriemia (piel y partes blandas, heridas quirúrgicas o catéteres), antecedente de estancia previa en Unidad de Cuidados Intensivos (UCl) y adquisición nosocomial o comunitaria de la infección. La infección se definió como de adquisición comunitaria si SA se aislaba en las primeras 48 horas de ingreso del paciente y éste no había ingresado en el mes previo. Se consideró que la bacteriemia era de adquisición nosocomial en los casos en los que el hemocultivo positivo se obtuvo a partir del tercer día de hospitalización en pacientes que al ingreso no presentaban signos o síntomas de infección y también en los casos en que el hemocultivo se obtuvo antes del segundo día de hospitalización, pero el paciente tenía antecedentes de ingreso hospitalario en el último mes.

Se definió la bacteriemia estafilocócica como la presencia de SA en un hemocultivo o más. Cada episodio de bacteriemia se evaluó siguiendo las recomendaciones habituales mediante la extracción de dos muestras de sangre;las muestras se inocularon en frascos de hemocultivos y se incubaron durante 5 días en un sistema automatizado (BACTEC 9240 System Beckton Dickinson Microbiology System).

El sistema MicroScan ${ }^{\circledast}$ de DadeBehring y el método de microdilución en placa aplicando puntos de corte del Clinical and Laboratory StandardsInstitute (CLSI), permitieron la identificación bacteriana de SA y la determinación de la sensibilidad in vitro.Las características clínicas y epidemiológicas de los pacientes se estudiaron mediante medida de tendencia central (porcentajes). Para el estudio de los factores de riesgo y pronósticos, se realizó análisis bivariante (Chi cuadrado de Pearson y Test de Fisher) y multivariante (regresión logística). El análisis estadístico de los datos se realizó con la herramienta informática SPSS versión 15.0 para Windows.

\section{RESULTADOS}

Se estudiaron de 57 casos, las bacteriemias producidas por SA, de los cuales $36(63,15 \%)$ fueron causadas por SAMS y 21 (36,84\%) por SARM. Las características clínicas y epidemiológicas de los pacientes con bacteriemia por SA se muestran en la tabla 1.

Las bacteriemias por SA fueron más frecuentes en los Servicios Médicos (39 casos, 68,42\%) que en los Servicios Quirúrgicos (10 casos, 17,54\%) y que en el resto de Servicios estudiados (8 casos, $14,03 \%)$. En cuanto al patrón de resistencia antibióticaen nuestro estudio, todas las cepas fueron sensibles in vitro a vancomicina, con valores de CMI (concentración mínima inhibitoria) $\leq 2 \mathrm{mg} / \mathrm{L}$ en todos los aislamientos. Tampoco se encontró alguna cepa resistente a linezolid ni a teicoplanina. En la tabla 2 se muestra el análisis bivariado de los factores de riesgo asociados a la resistencia a meticilina entre los pacientes con bacteriemia por SA evidenciándose que existen algunos factores significativamente asociados a esta resistencia: edad mayor de 65 años, antibioterapia previa y adquisición nosocomial de la infección. Los resultados del análisis de regresión logística realizado a aquellas variables que resultaron potenciales factores de riesgo en el análisis bivariado se presentan en la tabla 3. Para cada variable se muestra Odds Ratio y sus intervalos de confianza al 95\%. De este análisis pudo observarse que los factores de riesgo que explicaron la resistencia a meticilina en los pacientes estudiados fueron los mismos que en el estudio bivariado: edad mayor de 65 años, antibioterapia previa y adquisición nosocomial de la infección. Comparando porcentaje de bacteriemias por SARM $(43 \%)$ y SAMS $(56,4 \%)$, las ocasionadas por cepas sensibles a meticilina fueron más frecuentes que las producidas por SARM en todos los servicios de hospitalización, aunque no se encontraron diferencias estadísticamente significativas. En cuanto a la mortalidad, fue del $28,57 \%$ en los pacientes 
Tabla 1. Características clínicas y epidemiológicas de 57 pacientes con bacteriemia por $S A$.

\begin{tabular}{|c|c|c|}
\hline Características & $\mathbf{n}$ & $\%$ \\
\hline Edad $\geq 65$ años & $45 / 57$ & 78,95 \\
\hline Sexo masculino & $39 / 57$ & 68,42 \\
\hline Exitus & $19 / 57$ & 33,33 \\
\hline Diabetes & $16 / 57$ & 28,07 \\
\hline Neoplasia & $18 / 57$ & 31,58 \\
\hline Enfermedad pulmonar obstructiva crónica & $8 / 57$ & 14,04 \\
\hline Insuficiencia renal & $15 / 57$ & 26,32 \\
\hline Catéter & $7 / 57$ & 12,28 \\
\hline Cirugía & $11 / 57$ & 19,30 \\
\hline Antibioterapia previa & $19 / 57$ & 33,33 \\
\hline Ingreso último año & $30 / 57$ & 52,63 \\
\hline Foco infección & $34 / 57$ & 59,65 \\
\hline Adquisición nosocomial & $27 / 57$ & 47,37 \\
\hline SARM & $21 / 57$ & 36,84 \\
\hline Estancia previa en Unidad Cuidados Intensivos & $8 / 57$ & 14,04 \\
\hline n: Número de pacientes & & \\
\hline
\end{tabular}

Tabla 2. Análisis bivariado de factores asociados a la resistencia a meticilina en pacientes con bacteriemia por $S A$

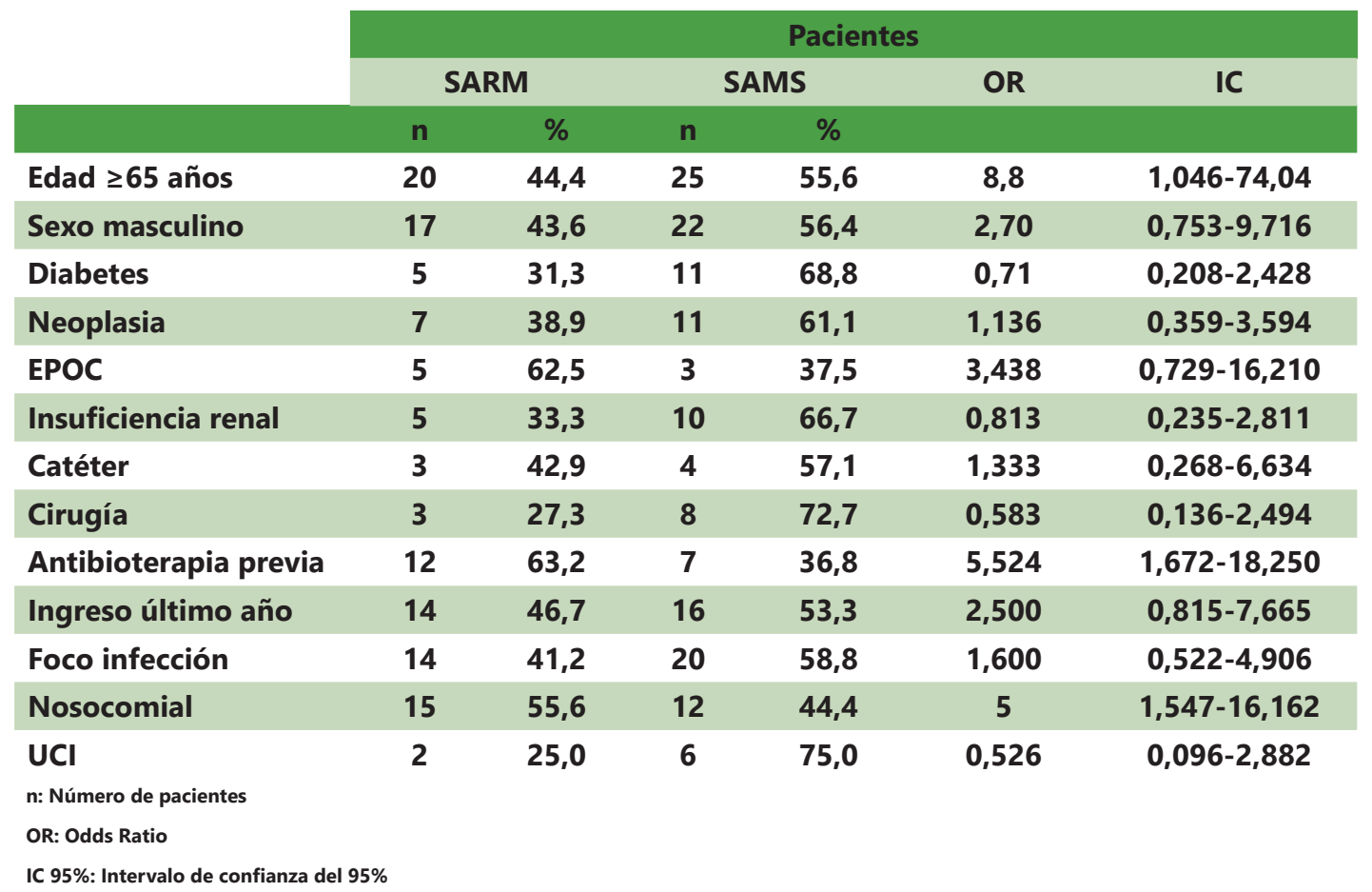

con bacteriemia por SARM y del $36,11 \%$ en los pacientes por SAMS.

Los factores de riesgo de mortalidad de los pacientes con bacteriemia por SA que se incorporaron en el estudio bivariado fueron los mismos que en el estudio de asociación con resistencia a meticilina, añadiéndose a este análisis la presencia de SARM. Ninguna de las variables investigadas resultó estadísticamente significativa en el estudio bivariante (Tabla 4). El $71,43 \%$ de las cepas de SARM fueron resistentes a eritromicina, el $33,33 \%$ a clindamicina y el 
Tabla 3. Resultados análisis multivariante de los factores de riesgo de bacteriemia por SARM

\begin{tabular}{|c|c|c|c|c|}
\hline \multirow{2}{*}{$\begin{array}{l}\text { Variables Incluidas } \\
\text { en el modelo }\end{array}$} & \multirow{2}{*}{$\mathbf{p}$} & \multirow{2}{*}{$\operatorname{Exp}(B)$} & \multicolumn{2}{|c|}{ I.C. $95,0 \%$ para EXP(B) } \\
\hline & & & Inferior & Superior \\
\hline Edad > 65 & 43 & 10,849 & 1,079 & 109,123 \\
\hline Antibiótico previo & 31 & 4,420 & 1,145 & 17,067 \\
\hline Adquisición nosocomial & 32 & 4,192 & 1,128 & 15,581 \\
\hline Constante & 2 & 21 & & \\
\hline
\end{tabular}

Tabla 4. Análisis bivariado de factores asociados a mortalidad entre los pacientes con bacteriemia por SA

\begin{tabular}{|lcccc|}
\multicolumn{1}{c}{ Variables } & Exitus & & & \\
& SI & NO & IC \\
\hline Edad $\geq 65$ años & 16 & 29 & 1,422 & $0,495-4,089$ \\
\hline Sexo masculino & 12 & 27 & 0,791 & $0,375-1,669$ \\
\hline Diabetes & 5 & 11 & 0,915 & $0,394-2,124$ \\
\hline Neoplasia & 7 & 11 & 1,264 & $0,599-2,666$ \\
\hline EPOC & 3 & 5 & 1,148 & $0,431-3,062$ \\
\hline Insuficiencia renal & 5 & 10 & 1,000 & $0,434-2,302$ \\
\hline Catéter & 1 & 6 & 0,397 & $0,062-2,528$ \\
\hline Cirugía & 3 & 8 & 0,784 & $0,276-2,225$ \\
\hline Antibioterapia previa & 6 & 13 & 0,923 & $0,417-2,045$ \\
\hline Ingreso último año & 9 & 21 & 0,810 & $0,388-1,690$ \\
\hline Foco infección & 8 & 26 & 0,492 & $0,234-1,032$ \\
\hline Nosocomial & 8 & 19 & 0,808 & $0,383-1,707$ \\
\hline UCI & 3 & 5 & 1,148 & $0,431-3,062$ \\
\hline SARM & 6 & 15 & 0,791 & $0,354-1,768$ \\
\hline
\end{tabular}

RR: Riesgo Relativo

IC 95\%: Intervalo de confianza del 95\%

95,24\% a ciprofloxacino. No hubo ningún aislado resistente a vancomicina ni teicoplanina.

\section{DUSCUSIÓN}

En la investigación, durante el período de análisis se observó una mortalidad del 33,3\% en las bacteriemias estafilocócicas, siendo la mortalidad asociada a la resistencia a la meticilina del 28,57\%, porcentaje semejante al de la mayoría de estudios publicados en la literatura ${ }^{14}$. El impacto de la resistencia a meticilina sobre su contribución a la mortalidad ha sido tradicionalmente un tema controvertido $^{15}$. Dado que las infecciones por microorganismos multirresistentes ocurren con frecuencia en pacientes con enfermedades graves, antecedentes de hospitalizaciones prolongadas y uso previo de antibióticos ${ }^{16,17}$, resulta difícil atribuir la mortalidad a la propia virulencia del SARM. Como señalan diferentes autores, la resistencia a meticilina no condicionaría por sí misma el pronóstico de estas infecciones, sino que influirían además otros factores como la situación de base del paciente o la administración de tratamientos empíricos lo más adecuados posibles ${ }^{18}$. En nuestro estudio, la mortalidad de los pacientes por bacteriemia por SAMS fue mayor que en aquellos con SARM y la sensibilidad/ resistencia a meticilina no se asoció de modo estadísticamente significativo a la mortalidad. La relación estadísticamente significativa encontrada en nuestro trabajo entre resistencia a meticilina y factores de riesgo como edad mayor de 65 años, antibioterapia previa y 
adquisición nosocomial de la infección, también se han descrito como factores predisponentes para la adquisición de estas cepas ${ }^{19}$. De acuerdo con nuestros datos y desde un punto de vista epidemiológico en nuestra área sanitaria, debería considerarse que puede haber resistencia a meticilina en las bacteriemias de pacientes con alguno o varios de los factores anteriormente descritos. SARM, además de presentar resistencia intrínseca a penicilinas y cefalosporinas, presenta mayores niveles de resistencia que SAMS a otros antimicrobianos ${ }^{20}$, lo que limita en gran medida las alternativas terapéuticas. A esto hay que añadirla aparición de cepas con resistencia a glicopéptidos en diversas regiones del mundo, incluida Europa. Los aislados GISA o VISA se asocian a fracasos terapéuticos con vancomicina ${ }^{21}$. En nuestro estudio, todas las cepas de SA fueron sensibles a vancomicina y teicoplanina. Afortunadamente todavía no hemos detectado cepas VISA en nuestro medio,aunque sí hemos constatado el mayor patrón de resistencia de SARM frente a SAMS, como describen otros autores ${ }^{2}$. Según el Estudio de Vigilancia de Resistencia a los Antimicrobianos (VIRA) ${ }^{22}$, la resistencia de SARM en 2010 a eritromicina se situó en un 55,6\%, siendo el $71,43 \%$ en nuestra serie. En cuanto a la resistencia a clindamicina, el estudio VIRA la fija en un $15 \%$, siendo nuestro porcentaje también superior (33,33\%). El porcentaje de resistencia a ciprofloxacino en nuestro medio también supera al descrito por el estudio VIRA. Las bacteriemias por SARM en nuestro Sector Sanitario representan el 36,84\% de las ocasionadas por SA atendidas en el ámbito hospitalario, porcentaje semejante al registrado en otras áreas de España.

La identificación del agente causal responsable de bacteriemiasy el estudio de sensibilidad en el laboratorio son esenciales para instaurar un tratamiento antibiótico adecuado y también para detectar precozmente resistencias como las recientemente descritas frente a los glucopéptidos ${ }^{23,24}$. La OMS recuerda que, aunque la resistencia es un proceso natural de adaptación por parte de los microorganismos cuando estos se exponen a los agentes antimicrobianos, se desarrolla mucho más rápidamente por el mal uso y el uso excesivo de estos fármacos. En este sentido, la OMS indica que el uso de antibióticos en los seres humanos ha aumentado en un $36 \%{ }^{13}$ entre 2000 y 2010 , siendo los países desarrollados los que tienen un mayor consumo por persona, aunque el consumo está creciendo rápidamente también en las economías emergentes. Por tanto, seleccionando de forma apropiada los antibióticos y evaluando adecuadamente los factores de riesgo que pueden favorecer la aparición de resistencia a meticilina, podremos disminuir la frecuencia de este problema clínico. Nuestro estudio debe interpretarse teniendo en cuenta sus limitaciones: el Hospital de Barbastro no es representativo de todos los hospitales de nuestro país y el número de casos estudiados es pequeño, lo que puede haber impedido encontrar algunas diferencias relevantes entre los grupos estudiados. No obstante, pensamos que los estudios locales, aunque con bajo número de aislamientos, nos permiten conocer el perfil epidemiológico de este tipo de infecciones en cada zona sanitaria para así adaptar estrategias de prevención y poder disminuir la incidencia de estas infecciones.

Consideramos que los hallazgos epidemiológicos encontrados en nuestro estudio (la asociación de bacteriemia por SARM a infección nosocomial, edad avanzada y uso previo de antibióticos) hacen recomendable que en los casos de sepsis grave se incluyan en el tratamiento empírico y antibiótico con actividad frente a SARM, ya que el tratamiento inicial adecuado, junto con la vigilancia epidemiológica, son fundamentales para disminuir la morbimortalidad de estas infecciones.

La incidencia de bacteriemia por SARM en nuestra zona sanitaria no difiere de la registrada en otras áreas de España. Las estrategias para disminuir la incidencia de estas infecciones y prevenir la adquisición de SARM en nuestro entorno serían tanto el control y uso adecuado de antimicrobianos, como la aplicación de programas de prevención de infecciones nosocomiales. En los pacientes con bacteriemia por SA, la resistencia a meticilina no se asocia a más mortalidad.Todos los aislamientos estudiados han sido sensibles in vitro avancomicina. 


\section{REFERENCIAS}

1. BannermanTL, PeacockSJ.Staphylococcus, Micrococcus, and other catalase-positive cocci.P.R. Murray, E.J. Baron, M.L. Landry, J.H. Jorgensen, M.A. Pfaller (Eds.), Manual of clinical microbiology (9th ed.), American Society for Microbiology, Washington, DC (2008), pp. 390-411. Disponible en: https:// www.scirp.org/(S(351jmbntvnsjt1aadkposzje))/reference/ ReferencesPapers.aspx?ReferencelD $=1757432$

2. Navascués A, García-Irure JJ, Guillén F. Situación de Staphylococcus aureus resistente a meticilina en el Hospital de Navarra (2000-2002). Anales Sis San Navarra [Internet]. 2004 [citado 2020 Enero 30] ; 27( 1 ): 21-25. Disponible en: http://scielo.isciii.es/scielo.php?script=sci_ arttext\&pid=S1137 66272004000100003\&lng=es

3. Cuevas O, Cercenado E, Vindel A, Guinea J, SánchezConde $M$, Sánchez-Somolinos $M$, et al. Evolution of the antimicrobial resistance of Staphylococcus spp. in Spain: Five nationwide prevalence studies, 1986 to 2002. Antimicrobial Agents and Chemotherapy. 2004;48(11):4240-5. Disponible en: http://aac.asm.org/cgi/ content/full/48/11/4240

4. Castellano González Maribel J, Perozo-Mena Armindo J. Mecanismos de resistencia a antibióticos $\beta$-lactámicos en Staphylococcus aureus. Kasmera [Internet]. 2010 [citado 2020 Enero 30]; 38( 1 ): 18-35. Disponible en: http:// ve.scielo.org/scielo.php?script=sci_arttext\&pid=S0075$52222010000100003 \& \operatorname{lng}=\mathrm{es}$

5. Sociedad Española de Medicina Preventiva Salud Pública e Higiene. EstudioEPINE: 1990-2014. [Internet] 2014 [Citado 2020 Enero 30] Disponible en: http://hws.vhebron. net/epine/Descargas/EPINE\%201990-2014\%20web.pdf

6. Jevons MP. Celbenin-resistant staphylococci. Br Med J. 1961;1:124-5. Disponible en: https://www.ncbi.nlm.nih. gov/pmc/articles/PMC1952888/

7. Fluit AC, Wielders CLC, Verhoef J, Schmitz FJ. Epidemiology and susceptibility of 3051 Staphylococcus aureus isolates from 25 university hospitals participating in the European SENTRY Study. J ClinMicrobiol. [Internet] 2001;39(10):3727-32. Disponible en: https://pubmed.ncbi. nlm.nih.gov/11574603/

8. Lodise TP, Graves J, Evans A, Graffunder E, Helmecke M, Lomaestro BM, et al. Relationship between Vancomycin MIC and Failure among Patients with MRSA Bacteremia Treated with Vancomycin. Antimicrob Agents Chemother. [Internet] 2008;52 (9):3315-20. Disponible en: https://aac. asm.org/content/52/9/3315

9. Hanaki H, Kuwuhara-Arai K, Boyle-Vavra S,Daum RS, Labischinski H, Hiramatsu K. Activated cell-wall synthesis is associated with vancomycin resistance in methicillinresistant Staphylococcusaureus clinical strains Mu3 and Mu50. J AntimicrobChemother. [Internet] 1998;42(2):199209. Disponible en: https://pubmed.ncbi.nlm.nih. gov/9738837/

10. Muto CA, John AJ, Ostrowsky BE, Richet HM, Jarvis WR, Boyce JM, et al. SHEA guideline for preventing nosocomial transmission of multidrug-resistant strains of Staphylococcus aureus and Enterococcus. Infect Control HospEpidemiol. [Internet] 2003;24(5):362-86. Disponible en: https://pubmed.ncbi.nlm.nih.gov/12785411/

11. Sopena N, Sabrià M. Staphylococcus aureus resistente a la meticilina. Med Clin (Barc). [Internet] 2002;118(17):6716. Disponible en: https://www.sciencedirect.com/science/ article/pii/S0025775302724901

12. Cercenado E, Sánchez-Carrillo C, Alcalá L, Bouza E y Grupo de Trabajo para el estudio de Estafilococos. Situación actual de la resistencia de Staphylococcus en España. Cuarto estudio nacional (1996). RevClin Esp. 1997;197 (Suppl 2):18-24. Disponible en: https://www.elsevier.es/esrevista-enfermedades-infecciosas-microbiologia-clinica28-articulo-staphylococcus-spp-espana-situacion-actualS0213005X0872706X

13. European Centre for Disease Prevention and Control (ECDC). Antimicrobial resistance surveillance in Europe. Annual report of the European Antimicrobial Resistance Surveillance Network (EARS-Net) [Internet] 2017. Disponible en: https://www.ecdc.europa.eu/en/ publications-data/surveillance-antimicrobial-resistanceeurope-2017

14. Castillo JS, Leal AL, Cortes JA, Álvarez CA, Sánchez R, Buitrago $G$ et al. Mortality among critically ill patients with methicillin-resistant Staphylococcus aureusbacteremia: a multicenter cohort study in Colombia. RevPanam Salud Pública. [Internet] 2012;32(5):343-50. Disponible en: https://iris.paho.org/handle/10665.2/9246

15. Cosgrove S, Sakoulas G, Perencevich E, Schwaber M, Karchmer AW, Carmeli Y. Comparison of mortalityassociา atedwithmethicillin-resistant and methicillin-susceptible Staphylococcus aureus bacteremia: a meta-analysis. Clin Infect Dis. [Internet] 2003;36(1):53-9. Disponible en: https://pubmed.ncbi.nlm.nih.gov/12491202/

16. European Centre for Disease Prevention and Control (ECDC). Summary of the latest data on antibiotic consumption in the European Union. Nov 2012. Disponible en: http://ecdc.europa.eu/en/eaad/Documents/ESAC-Netsummary-antibiotic-consumption.pdf.

17. Sanz P, Ramos A, Asensio A, García Ma J, Linares. M. Mortalidad y factores pronósticos en pacientes hospitalizados por bacteriemia adquirida en la comunidad. An Med Interna[Internet] 2006;23(2):66-72. Disponible en: http://scielo.isciii.es/scielo.php?script=sci_ abstract\&pid=S0212-71992006000200004

18. Whitby M, McLaws ML, Berry G. Risk of death from me $\neg$ thicillin-resistant Staphylococcus aureus bacteraemia: 
a meta-analysis. Med J Aust. [Internet] 2001;175(5): 264-7.Disponible en: https://pubmed.ncbi.nlm.nih. gov/11587259/

19. Blot S, Vandewoude K, Hoste E, Colardyn F. Outcome and attributable mortality in critically III patients with bacteremia involving methicillin-susceptible and methicillin-resistant Staphylococcus aureus. ArchlnternMed. 2002;162(19):2229-35. Disponible en: https://jamanetwork.com/journals/jamainternalmedicine/ fullarticle/213720

20. Cobo F, Manchado P, Porras J, Cárdenas A. Staphylococcus aureus resistente a la meticilina. Prevalencia actual en un área del sur de España. RevEspQuimioter. [Internet] 2002;15(3):264-7. Disponible en: https://dialnet. unirioja.es/servlet/articulo?codigo $=7361467$

21. Cantón R, Ruiz-Garbajosa P. Infecciones causadas por bacterias grampositivasmultirresistentes (Staphylococcus aureus y Enterococcus spp.). EnfermInfeccMicrobiolClin. [Internet] 2013;31(8):543-51. Disponible en: https://seimc. org/contenidos/documentoscientificos/eimc/seimc_ eimc_v31n08p543a551.pdf

22. Picazo JJ, Betriu C, Culebras E, Rodríguez-Avial I, Gómez M, LopezFabal F. Staphylococcus aureus resistente a meticilina: sensibilidad a la daptomicina a lo largo de un periodo de 10 años (2001-2010). RevEspQuimioter[Internet]2011;24(2):107-11. Disponible en: https://seq.es/seq/0214-3429/24/2/picazo.pdf

23. Guna MR, Larrosa N, Marín M, Rodríguez JC. Diagnóstico microbiológico de la bacteriemia y fungemia: hemocultivos y métodos moleculares. EnfermInfeccMicrobiolClin. [Internet] 2019;37(5):33540. Disponible en: https://seimc.org/contenidos/ documentoscientificos/procedimientosmicrobiologia/ seimc-procedimientomicrobiologia62.pdf

24. Menon V, Lahanas S, Janto C, Lee A. Utility of direct susceptibility testing on blood cultures: Is it still worthwhile?.J Med Microbiol. [Internet]2016;65(6):501-9. Disponible en: https://www.microbiologyresearch.org/ content/journal/jmm/10.1099/jmm.0.000259 